\title{
Percepción del usuario de la herramienta informática diseñada por el IESS para el reporte en línea de siniestros laborales.
}

\author{
Perception of the user of the computer tool designed by the IESS for the online report of \\ work accidents.
}

\begin{abstract}
Autora
Brenda Pilar Gamarra Zavala. Graduada de Maestría de Seguridad y Salud Ocupacional "Segunda Cohorte” de la Universidad San Gregorio de Portoviejo. Manabí.Ecuador.bripygaza@gmail.com https://orcid.org/0000-0002-8510-7071
\end{abstract}

Fecha de recibido: 2019-05-16

Fecha de aceptado para publicación: 2019-08-15

Fecha de publicación: 2019-09-30

\section{Resumen}

En Ecuador, el organismo responsable de las contingencias de accidentes de trabajo, es el Instituto Ecuatoriano de Seguridad Social (IESS), a través del Seguro especializado de Riesgos del Trabajo. Las prestaciones por accidentes de trabajo, inician con el reporte en el portal web. En el departamento de Riesgos del Trabajo de la provincia de Santo Domingo, se ha observado un número considerable de registros en línea con errores. El objetivo del estudio fue obtener la percepción del usuario, sobre la herramienta diseñada por el IESS para el registro de accidentes de trabajo. Se utilizó el método de encuesta, la herramienta fue un cuestionario original, aplicado a los responsables del registro en línea de accidentes laborales. Se obtuvo como resultados que un porcentaje considerable de responsables del registro en mención, perciben que la herramienta informática es "difícil de utilizar"; que un considerable porcentaje de los encuestados no cuentan con formación en Seguridad y Salud Ocupacional, y tampoco habían recibido capacitaciones por parte de Riesgos del Trabajo para el uso de la herramienta informática disponible en el portal web del IESS para este registro.

Palabras clave: Accidentes de trabajo; registro accidentes de trabajo IESS; registro de accidentes de trabajo Ecuador; registro en línea de accidentes de trabajo; riesgos del trabajo IESS.

\begin{abstract}
In Ecuador, the body responsible for the contingencies of occupational accidents is the Ecuadorian Institute of Social Security (IESS), through the specialized Occupational Risk Insurance. The benefits for accidents at work begin with the report on the web portal. In the department of Occupational Risks of the province of Santo Domingo, a considerable number of records have been observed in line with errors. The objective of the study was to obtain the user's perception of the tool designed by the IESS for the registration of occupational accidents. The survey method was used, the tool was an original questionnaire, applied to those responsible for the online registration of occupational accidents. The results obtained were that a considerable percentage of those responsible for the aforementioned registry perceive that the computer tool is "difficult to use"; that a considerable percentage of the respondents do not have training in Occupational Safety and Health, nor had they received training from Occupational Risks for the use of the computer tool available on the IESS website for this registry.
\end{abstract}

Keywords: Work accidents; IESS work accident record; registration of accidents at work Ecuador; online registration of occupational accidents; IESS work risks. 


\section{Introducción}

"El accidente de trabajo (AT) es todo suceso imprevisto y repentino que ocasiona al trabajador una lesión corporal o perturbación funcional, con ocasión o por consecuencia del trabajo que ejecuta por cuenta ajena" (Codigo del Trabajo, 2005, p. 91).

El organismo responsable de las contingencias de accidentes de trabajo en el Ecuador, es el IESS, a través del Seguro especializado de Riesgos del Trabajo, respecto del reporte de accidentes de trabajo está establecido "los formularios de aviso de accidente de trabajo, o de enfermedad profesional u ocupacional, disponibles en el portal web del IESS, deberán enviarse a través del sistema informático" (Resolución del IESS 513, 2016, p. 20).

En el departamento de Riesgos del Trabajo de Santo Domingo de los Tsáchilas se reciben mensualmente un promedio de 35 accidentes de trabajo, reportados por la parte empleadora, en el portal web del IESS, mediante la herramienta informática diseñada para el aviso de accidentes de trabajo.

Conjuntamente con el formulario de aviso se pueden presentar los documentos habilitantes para la calificación del siniestro, o incorporarlos al proceso dentro de los diez días laborables siguientes (Resolución del IESS 513, 2016). Estos siniestros, previo a calificarse como accidentes de trabajo, deben cumplir varios parámetros; se inicia con un reporte correcto en el portal web, seguido por la entrega de la documentación habilitante, en los tiempos establecidos.

De los 35 accidentes mensuales (promedio) receptados por el departamento de Riesgos del Trabajo de la provincia de Santo Domingo, aproximadamente 10 reportes presentan errores. La parte empleadora registra errores: en el tipo de accidente, fechas, lugar del accidente y en los diagnósticos. Estos errores en el registro retrasan el proceso de calificación e interfieren en la entrega de prestaciones a ser entregadas a los afiliados (trabajadores).

Cuando el empleador comete errores en el registro de accidente de trabajo, el médico ocupacional de Riesgos de Trabajo (profesional que formaliza la calificación) indica los cambios a ser realizados. Este proceso es sencillo, pero toma varios días y se retrasan los procesos.

Sumado a lo descrito, la parte empleadora debe entregar documentación que respalde el suceso, por ejemplo, todo accidente de trabajo, accidente de tránsito, debería contar con un documento que le brinde evidencia objetiva, tal es el caso del parte policial. Además, el empleador deberá presentar justificativos de horarios de trabajo, actividades realizadas por el trabajador, rutas de trabajo, entre otros.

Es importante señalar que previo a la emisión de la Resolución C.D.513, se encontraba vigente la Resolución C.D. 390, aprobada el 10 de noviembre de 2011; la cual en su disposición transitoria tercera, ya mencionaba la creación de aplicativos electrónicos para el reporte de siniestros laborales, y disponía que "hasta que se implementen los referidos aplicativos informáticos, los avisos de accidentes de trabajo y enfermedad profesional $\mathrm{u}$ ocupacional se presentarán en el formulario impreso en las unidades de riesgos del trabajo" (Resolución No. C.D.390, 2011, p. 28).

A partir del 6 de marzo del 2016, tras ser derogada la Resolución 390, entró en vigencia la Resolución C.D. 513, que en su artículo 43, en relación al aviso de accidente de trabajo, indica que los formularios de accidentes de trabajo, deben ser enviados a través del sistema informático del IESS, toda vez que se ha realizado el reporte en línea mediante el portal web 
del IESS, no es posible realizar cambios en caso de errores, sin realizar el proceso de solicitud antes mencionado.

En el Ecuador, un estudio realizado en el 2016 demostró que "por cada 100.000 trabajadores afiliados, la tasa de incidencia por accidentes de trabajo aumentó de 381,2 en el 2010 a 775,0 en el 2015" (Gómez, Algora, Bermúdez \& Vilaret, 2016, p. 171).

Es aún complejo lograr que las instituciones, además de dotar de equipos de protección personal (EPP) busquen mecanismos para bajar los índices de accidentabilidad y generen un ambiente de trabajo apropiado (Salvador Moreno, 2018).

En Ecuador no se cuenta con un estudio actualizado de incidencia de los últimos tres años, pero se debe mencionar que el autor tampoco encontró esta información actualizada sobre países vecinos. Pero en la página web del IESS, los casos de reporte van en ascenso cada año, lo cual, a criterio del autor, viene de la mano con un incremento en la presentación de avisos con errores. Respecto al registro de errores, en la herramienta del portal web, no se encontraron estudios previos; pero se obtuvieron datos de Colombia, el registro de accidentes de trabajo se realiza mediante las directrices emitidas en la Resolución 1570 del 2005 (Resolución número 1570 de 2005, 2005), la cual cuenta con un anexo técnico que describe el proceso a seguir, y pese a ello se presentan errores en el registro, los cuales serán enunciados.

Este estudio tiene como objetivo determinar la percepción que tiene la parte empleadora acerca de la herramienta informática diseñada para el registro en línea de accidentes de trabajo. El autor ha observado que existen dificultades con el uso de la herramienta informática para el reporte de accidentes de trabajo, lo que motiva la realización de este estudio, ya que "identificar problemas relevantes de la práctica e investigarlos, fundamentar los resultados obtenidos, publicarlos y defenderlos son momentos esenciales del quehacer científico" (Borroto Cruz, 2013, p. 1).

Como objetivos específicos se pretende obtener datos que permitan inferir si la percepción del usuario está relacionada con los conocimientos en seguridad y salud ocupacional y/o, con las capacitaciones recibidas por parte de Riesgos del Trabajo.

Los resultados obtenidos, servirán de base para nuevos estudios que permitan diseñar estrategias o propuestas para llegar de manera adecuada a la parte empleadora, y disminuir los errores de los registros en línea de siniestros, de igual manera disminuir los tiempos de entrega de documentación habilitante para la calificación. Y así asegurar el cumplimiento en la entrega de las prestaciones básicas de protección del seguro de Riesgos del Trabajo (Congreso Nacional, 2017).

\section{Metodología}

Se trata de una investigación exploratoria y de corte transversal. Según (Hernández Sampieri, Fernández \& Baptista, 2010) "los estudios exploratorios se efectúan, normalmente, cuando el objetivo es examinar un tema o problema de investigación poco estudiado, del cual se tienen muchas dudas o no se ha abordado antes". Se utilizó el método de encuesta. Se aplicó una herramienta original preparada con la colaboración de un psicólogo y un ingeniero en maestría de Seguridad y Salud Ocupacional.

La muestra estuvo representada por el $100 \%$ de medianas y grandes empresas de la Provincia de Santo Domingo de los Tsáchilas, cuyos responsables de registrar en línea los accidentes de trabajo, acudieron al departamento de Riesgos del Trabajo de la Provincia de Santo Domingo en los meses de enero y febrero del año 2019 y que accedieron a llenar el 
cuestionario. Además, se incluyeron responsables del registro de AT, que acudieron a las capacitaciones dictadas por Riesgos del Trabajo a las empresas en los meses de enero y febrero del 2019. Cabe indicar que no se incluyeron microempresas.

El personal involucrado fue la alta gerencia, responsables de talento humano, profesionales de las áreas de seguridad y salud en el trabajo, y todos aquellos que formaban parte del equipo responsable de registrar los siniestros laborales que ocurren en las medianas y grandes empresas. Todas las personas incluidas en el estudio, conocen y han manejado la herramienta del portal web del IESS para el reporte de Accidentes de Trabajo.

Previo al llenado de la herramienta, se explicó que sus respuestas serían tratadas con total confidencialidad, y que la información recopilada sería utilizada estrictamente para fines investigativos. En los resultados no se mencionan nombres de empresas ni personas.

Es meritorio mencionar que se efectuó una prueba piloto, en la cual la herramienta constaba de 20 preguntas, las cuales fueron percibidas por los encuestados como complejas, y existían preguntas que se enfocaban más en determinar si el encuestado conocía la normativa legal y no en la percepción del mismo sobre la herramienta informática; por lo cual solo fue aplicada a 15 personas. Una segunda encuesta, ya modificada y con 18 preguntas, fue aplicada a 12 personas, pero se mantenía la complejidad, y los usuarios tenían dudas de como contestar, por lo cual se revisó y modificó nuevamente realizando énfasis en la percepción del usuario, que es el objetivo del estudio.

El constructo final, consta de quince preguntas, con criterio de selección.

Fueron consideradas los siguientes elementos:

1. Área de la empresa a la que pertenece: se consideró esta variable, con el objetivo de evidenciar, quienes son los responsables en las medianas y grandes empresas de la provincia, de reportar en línea los Accidentes de Trabajo.

2. Formación o adiestramiento en seguridad y salud ocupacional: esta variable, buscó demostrar si los responsables del registro en línea de accidentes de trabajo, tenían competencias en el área de seguridad y salud ocupacional.

3. Capacitaciones de Riesgos del Trabajo: con esta variable se obtuvieron datos que indican si los responsables del registro en línea de Accidentes Laborales, habían recibido capacitaciones por parte de Riesgos del Trabajo, sobre cómo realizar este registro.

4. Lugar del accidente: en la herramienta informática para el registro de Accidentes de Trabajo, existe una sección en la cual el empleador debe seleccionar el tipo de accidente; entre las opciones están: "en el centro o lugar de trabajo habitual", "en otro centro o lugar de trabajo", "en comisión de servicios", "en desplazamiento de la jornada laboral e "in itinere".

En la herramienta aplicada se detallaron cinco tipos de accidentes basados en casos similares a los recibidos en el área de Riesgos del Trabajo de Santo Domingo. El usuario debía indicar a qué tipo de accidente correspondía cada ejemplo. Estas preguntas (cinco en total, una por cada tipo "Lugar del accidente"), fueron agrupadas en una sola variable, y se consideró para la tabulación y análisis de datos dos tipos de valores, la primera corresponde a que todas las respuestas fueron respondidas correctamente, y el otro valor corresponde a que hubo errores en una o más respuestas.

5. Documentación habilitante: se trata de toda la documentación que la parte empleadora debe entregar en riesgos del trabajo, para respaldar el siniestro reportado. Entre ellos, horarios de trabajo, funciones del trabajador accidentado, parte policial de ser el caso, entre otros. Con esta variable, se buscó evidenciar la percepción del usuario al responder si la herramienta indica qué documentación de respaldo se debe presentar en cada tipo de accidente. Se realizaron cinco preguntas, una por cada tipo de accidente, y se agruparon en una sola 
variable; los valores considerados fueron tres, el primero corresponde a que el usuario percibe que en todos los tipos de accidente la herramienta indica la documentación habilitante, el segundo valor indica que no en todos los casos, y el tercer valor, que en ninguno de los casos.

6. Mayor dificultad: esta variable mide la sección de la herramienta informática para registros de accidentes laborales, percibida como la que genera mayor dificultad al momento de registrar un nuevo accidente laboral en las empresas incluidas. Se incluyeron: "Horario de trabajo", "Tipo de accidente", "Hora del accidente", "Fecha de accidente", "Diagnóstico" y "Descripción del accidente".

7. Uso de la herramienta: esta variable se incluyó para que los usuarios encuestados expresen cómo perciben el uso de la herramienta informática para el registro de accidentes. Las dimensiones fueron: "fácil", "ni fácil, ni difícil, "difícil".

Las preguntas fueron elaboradas basadas en la Resolución C.D. 513 del IESS y en la herramienta del portal web del IESS, considerando que éstas fuesen llenadas por personal perteneciente al equipo responsable de la notificación en línea de Accidentes Laborales en las empresas; con la aspiración de recolectar información sobre su formación en seguridad y salud ocupacional, el conocimiento básico de la normativa C.D.513, y relacionarlo con la percepción de los usuarios.

El departamento de Riesgos del Trabajo, también imparte capacitaciones en materia de Seguridad y Salud Ocupacional dirigidas a las empresas; se aprovecharon estas capacitaciones para al final de las mismas, pedir a los responsables del registro en línea de accidentes la colaboración en el llenado de la encuesta.

Una vez obtenidas las encuestas respondidas, 62 en total, se procedió a ingresar los datos en el programa de análisis estadístico SPSS, que se describe como "el software estadístico líder mundial para empresas, gobierno, organizaciones de investigación y académicas" (Valencia \& Cuevas Romo, s.f).

\section{Resultados}

Inicialmente se pretendió abarcar una muestra mayor, sin embargo, se limitó en función de la asistencia de las partes involucradas a Riesgos del Trabajo. Cabe mencionar que no existen otros estudios en el país, por lo que estuvo limitada la relación o comparación de resultados.

El autor, argumenta la importancia del estudio efectuado, ya que toda vez que se presenta un accidente de trabajo, el registro en línea de este suceso inicia con el uso de la herramienta informática diseñada para el efecto, y si el usuario tiene dificultades o errores en el uso de la misma, los procesos no se ejecutan de manera óptima. Cabe resaltar que, en el país, no se dispone de otra herramienta para registrar siniestros laborales, considerando la importancia de comprender la misma. Del estudio realizado, sobre la percepción del usuario acerca de la herramienta informática, los resultados más relevantes fueron:

Respecto a la percepción de la dificultad de la herramienta, existe un alarmante porcentaje de usuarios $(64,5 \%)$, que considera que la herramienta no es amigable, lo cual da aval a la realización de este estudio. (Ver Tabla 1 en Anexos).

En relación a la documentación habilitante, se obtiene que el $45.2 \%$ de usuarios percibe que la herramienta no indica en ninguno de los tipos de accidente, la documentación habilitante para su calificación. Lo cual representa una cifra alarmante si consideramos que sólo el $12.9 \%$ percibe que la herramienta indica la documentación habilitante en todos los casos. (Ver Tabla 2 en Anexos). 
Los resultados sobre la percepción en relación a la sección más difícil, se obtiene que la parte empleadora en un 79\% percibe como la sección más difícil, el registro del diagnóstico. (Ver Tabla 3 en Anexos).

El constructo empleado, fue diseñado además para valorar aspectos que se consideran determinantes al momento de registrar un siniestro laboral; entre ellas obtener el porcentaje de usuarios encuestados con formación y/o adiestramiento en SSO, de lo cual se obtuvo como dato relevante que el $29 \%$, no tiene formación en SSO, lo cual cobra importancia al relacionarlo con el porcentaje de encuestados que ha recibido capacitaciones por parte de Riesgos del Trabajo para el Registro de siniestros laborales, donde el $66.1 \%$ de encuestados no han recibido estas capacitaciones.

\section{Discusión}

De los resultados obtenidos llama la atención que del total de los encuestados el $66.1 \%$ no ha recibido capacitaciones por parte de Riesgos del Trabajo, sobre cómo realizar el registro en línea de los AT, cuestión contradictoria, dado que existe un cronograma mensual de capacitaciones dirigidas a las empresas en todas las provincias al igual que en la Provincia de Santo Domingo de los Tsáchilas, las cuales son dirigidas a los representantes legales y personal de Seguridad y Salud Ocupacional vía correo electrónico. Se puede inferir que las empresas no están acudiendo a las capacitaciones o en su defecto, no están acudiendo las personas responsables del registro de accidentes de trabajo. A lo descrito se suma que, en las medianas y grandes empresas de Santo Domingo de los Tsáchilas, existe un porcentaje considerable de reportes de Accidentes de Trabajo, y que son efectuados por personas que no tienen formación o adiestramiento en Seguridad y Salud Ocupacional, lo que conlleva al autor a inferir que no conocen de la normativa legal vigente y por ello registran errores al momento de la notificación.

Al analizar la variable que evidencia si la herramienta indica la documentación habilitante, el $41.2 \%$ expresa que en algunos casos si y en otros no, y el $45.2 \%$ expresa que en ninguno de los casos; ambas respuestas indican que el usuario percibe que la herramienta no ofrece la información completa al momento de registrar un accidente de trabajo; por lo que el autor infiere que el usuario (parte empleadora), no está explorando adecuadamente la herramienta informática y sus anexos, ya que en uno de ellos se puede encontrar la documentación básica a presentar en los accidente de trabajo.

Otro de los resultados importantes es la percepción de dificultad de la herramienta, la cual es considerada para el mayor porcentaje (64.5\%), como difícil, lo cual podría estar en relación al desconocimiento de la normativa y a la falta de capacitación, resultado que demuestra la importancia y e influencia que tiene el Departamento de Riesgos del Trabajo, en la capacitación dirigida a la parte empleadora.

Es necesario mencionar sobre el mecanismo de notificación de accidentes de trabajo en países vecinos, por ejemplo, en Colombia, el empleador notifica el accidente de trabajo a la entidad de salud en que se encuentre afiliado el trabajador, además a la correspondiente Administradora de Riesgos Laborales (ARL) y a la Dirección Territorial u Oficina Especial del Ministerio del Trabajo donde haya sucedido el accidente (Resolución número 02851, 2015). En este país la resolución 1570, en relación a los reportes de los AT, considera que "las variables, datos, mecanismos de recolección y envío de la información que las entidades administradoras de riesgos profesionales, entidades promotoras de salud y juntas de calificación de invalidez, deben remitir a la Dirección General de Riesgos Profesionales del Ministerio de la Protección Social" (Resolución número 1570, de 2 de junio, 2005). 
Adicionalmente, se instituye sanciones por faltantes en la información (errores). Respecto de los formatos establecidos para este registro, están determinados en la resolución 0156 (Resolución número 0156, de 27 de enero, 2005).

Cabe expresar que Colombia, también presenta errores de registro de accidentes de trabajo tal como lo indica un estudio realizado en una empresa de aves en el 2010, en el cual consta que todos los reportes previos a ser notificados en la ARP (administradora de riesgos profesionales, actualmente ARL), eran revisados por el encargado de Salud Ocupacional, y que en esta etapa se identificaron inconsistencias en la descripción de los accidentes, en los datos del trabajador, diagnósticos y en el centro de trabajo. Así mismo expresa que en algunos casos los registros se entregaban extemporáneamente (Torres Martínez, 2010). Este estudio coincide con los resultados obtenidos, pese a que en este país el sistema de registro está a criterio del autor, mejor estructurado, ya que las ARL, podrían fungir como asesoras.

En México, desde el 1ero de enero del 2016, entró en vigencia el "Acuerdo por el que se crea el sistema de avisos de accidentes de trabajo y se dan a conocer los formatos para informar los accidentes y defunciones de los trabajadores" (SEGOB, 2015) así, la Secretaría del Trabajo y Previsión Social dispuso en línea el Sistema de Avisos de Accidentes de Trabajo (Gobierno de México, 2018) en este sistema se encuentra disponible el formato para el reporte de los accidentes de trabajo con los respectivos instructivos, con la consecuente opción patrono/empleador de entregar los reportes en físico.

Si se realiza una comparación entre la página del registro de México y Ecuador (IESS) ambas cuentan con tutoriales de cómo registrar un accidente de trabajo; no obstante, en el Ecuador la diferencia radica que el empleador tiene solo la opción de registrar en línea; lo que conlleva a inferir que los responsables del registro de accidentes en nuestro país, no están explorando adecuadamente la página web, o que esta es muy compleja y no permite que la parte empleadora localice la información, lo que denota la falta de capacitación.

En Perú los registros se llevan a cabo de manera muy similar al Ecuador. El vecino país tiene vigente el "Sistema de Información para el Registro Único de Accidentes de Trabajo y Enfermedades Ocupacionales (SAT), que es una iniciativa del Ministerio de Trabajo y Promoción del Empleo (MTPE). Mediante este sistema las empresas y centros médicos pueden reportar incidentes y accidentes de trabajo" (Gobierno, 2018). A diferencia de Ecuador, en Perú, se involucra a la parte médica, en el registro de accidentes laborales, lo cual representa un aspecto positivo, ya que se minimizan los errores al momento del registro, lo cual se infiere basada en que en este país no se encontró bibliografía relacionada a dificultades con el sistema de Registros de accidentes de trabajo.

\section{Conclusiones}

Los datos obtenidos reflejan que el usuario (empleador) tiene la percepción de que la herramienta para el registro de AT es "difícil", que no especifica la documentación a presentar como habilitante, y que, además, la sección con mayor dificultad al momento del registro, es el diagnostico. De manera general se concluye que el usuario percibe la herramienta como "no adecuada, difícil".

Se debe considerar junto a los resultados anteriores, que un $29 \%$, no cuenta con formación o adiestramiento en SSO, lo cual lleva inferir que existe una relación entre registros inadecuados y falta de formación.

Respecto a las capacitaciones por parte de Riesgos del Trabajo, acerca de cómo realizar el registro en línea de los AT, el $66.1 \%$ de los responsables del registro mencionado, no han 
recibido capacitaciones, lo cual permite inferir una relación aún más directa entre los registros inadecuados y la falta de capacitación sobre el uso de la herramienta informática para el registro de los AT.

De manera general se concluye que los errores cometidos por la parte empleadora al momento de registrar un nuevo accidente de trabajo, están determinados por la falta de capacitación en el uso de la herramienta informática, la falta de preparación en SSO, y a la complejidad de la herramienta, esta última a criterio del autor es percibida de esta manera por falta de conocimiento del usuario.

Finalmente, considerando que no es posible controlar si los usuarios de la herramienta informática están capacitados para el registro adecuado de siniestros laborales en línea, mediante la misma; ésta debería ser analizada, poniendo énfasis en la sección del diagnóstico, que es la que mayor dificultad causa a los usuarios y su contenido podría estructurarse de manera que resulte más llamativa y fácil de utilizar al usuario.

\section{Referencias Bibliográficas}

Borroto Cruz, E. R. (2013). Investigar, publicar y debatir para transformar. Educación Médica Superior., 27(1), 1-3.

Código del Trabajo. (2005). 91. ECUADOR: Lexis.

Congreso Nacional. (12 de Diciembre de 2017). Ley de Seguridad Social. Obtenido de http://www.trabajo.gob.ec/wp-content/uploads/2018/03/12.-Ley-de-Seguridad-Social.pdf.

Gobierno. (2018). gob.pe. Obtenido de Plataforma digital única del Estado Peruano: https://www.gob.pe/774-notificar-accidentes-en-el-trabajo-y-enfermedades-ocupacionales

Gobierno de México. (2018 de Enero de 2018). El portal único del gobierno. Obtenido de www.gob.mx: https://www.gob.mx/

Gómez García, A., Algora Buenafé, A., Bermúdez, S., \& Vilaret Serpa, A. (2016). Notificación de Accidentes de Trabajo y Posibles Enfermedades Profesionales en Ecuador, 2010-2015. Ciencia \& Trabajo, 18(57), 166-172. Obtenido de www.cienciaytrabajo.cl

Hernández Sampieri, R., Fernández Collado, C., \& Baptista Lucio, P. (2010). Metodología de la investigación (Vol. 3). . México.: McGraw-Hill.

Reglameto del Seguro General de Riesgos del Trabajo. (10 de noviembre de 2011). 26-27. Quito, Distrito Metropolitano, Ecuador.

Resolución del IESS 513. (04 de marzo de 2016). Reglamento el Seguro General de Riesgos del Trabajo. Registro Oficial Edición Especial 632(articulo 43). Quito, Ecuador.

Resolución número 0156, de 27 de enero. (2005). Por la cual se adoptan los formatos de informe de accidente de trabajo y de enfermedad profesional y se dictan otras disposiciones (2005). Colombia. 
Resolución número 02851. (2015). Por la cual se modifica el artículo $3^{\circ}$ de la Resolución número 156 de 2005 (2015). Colombia.

Resolución número 1570 de 2005. (2005). Colombia.

Resolución número 1570, de 2 de junio . (2005). Por la cual se establecen las variables y mecanismos para recolección de información del Subsistema de Información en Salud Ocupacional y Riesgos Profesionales y se dictan otras disposiciones, Diario Oficial No. 45.927 . Colombia.

Salvador Moreno, J. E. (2018). Riesgos Psicosociales del Sector Aeroportuario de Manta. Revista San Gregorio, 30-39.

SEGOB. (14 de 12 de 2015). Acuerdo por el que se crea el Sistema de Avisos de Accidentes de Trabajo y se dan a conocer los formatos para informar los accidentes y defunciones de los trabajadores. Obtenido de Diario Oficial de la Federación: http://dof.gob.mx/nota_detalle.php?codigo $=5419853 \&$ fecha $=14 / 12 / 2015$

Torres Martínez, M. A. (2010). Gestion de las actividades de salud ocupacional del area comercial de Avidesa MACPOLLO S.A. 55-56-57. Bucaramanga. Obtenido de https://repository.upb.edu.co/handle/20.500.11912/1002

Valencia, S. M. (s.f.). Manual introductorio al SPSS Statistics Standard . Edition 22 (Doctoral dissertation, Instituto Politécnico Nacional). 


\section{Anexos}

Tabla 1.- Considera que el uso la herramienta informática para el registro de accidentes de trabajo es:

\begin{tabular}{|l|l|l|l|}
\hline \multicolumn{2}{|c|}{} & Frecuencia & Porcentaje \\
\hline Válido & Fácil. & 7 & 11,3 \\
\cline { 2 - 4 } & Ni fácil, ni difícil. & 15 & 24,2 \\
\cline { 2 - 4 } & Difícil & 40 & 64,5 \\
\cline { 2 - 4 } & Total & 62 & 100,0 \\
\hline
\end{tabular}

Tabla 2.- La herramienta indica la documentación para calificación de siniestros

\begin{tabular}{|l|l|l|l|l|}
\hline \multicolumn{2}{|l|}{} & Frecuencia & Porcentaje & Porcentaje válido \\
\hline Válido & $\begin{array}{l}\text { Sí, en todos los tipos de } \\
\text { accidente. }\end{array}$ & 8 & 12,9 & 12,9 \\
\cline { 2 - 5 } & Unos Sí y otros No. & 26 & 41,9 & 41,9 \\
\cline { 2 - 5 } & No, ningún tipo de accidente & 28 & 45,2 & 45,2 \\
\cline { 2 - 5 } & Total & 62 & 100,0 & 100,0 \\
\hline
\end{tabular}

Tabla 3.-Sección de la herramienta que genera mayor dificultad

\begin{tabular}{|l|l|l|l|l|}
\hline \multicolumn{2}{|c|}{} & Frecuencia & Porcentaje & Porcentaje válido \\
\hline Válido & Horario de trabajo & 1 & 1,6 & 1,6 \\
\cline { 2 - 5 } & Tipo de accidente & 11 & 17,7 & 17,7 \\
\cline { 2 - 6 } & Diagnostico & 49 & 79,0 & 79,0 \\
\cline { 2 - 5 } & Descripción del accidente & 1 & 1,6 & 1,6 \\
\cline { 2 - 5 } & Total & 62 & 100,0 & 100,0 \\
\hline
\end{tabular}

